BIJDRAGEN TOT DE KENNIS DER FAUNA VAN CURAÇAO.

Resultaten eener reis van Dr. C. J. VAN DER HORST in 1920.

\title{
WESTINDISCHE SIPUNCULIDEN UND ECHIURIDEN
}

VON

\author{
ADA TEN BROEKE.
}

(Mit 25 Textfiguren).

Die von Dr. C. J. van DER HoRst in Amsterdam im Jahre 1920 von seiner Reise nach Curaçao heimgebrachten Gephyreen lieferten das Material für diese Untersuchungen. Nur einige wenige Arbeiten handeln über Westindische Gephyreen, unter denen ich in erster Reihe die Arbeit FISCHER's über das von Prof. Kükenthal und Prof. Hartmeyer gesammelte Material hervorheben will. Diese Sammlung enthielt 10 Sipunculiden und 2 Echiuriden, darunter vier neue Arten. Es fanden sich:

Siphonosoma occidentale Spengel, Physcosoma antillarum Grube, Ph. dentigerum Selenka und de Man, Ph. nigrescens Keferstein, Ph. pectinatum Keferstein, Ph. varians Keferstein, Aspidosiphon speciosus Gerould, A. spinoso-scutatus Fischer, A. steenstrupi Diesing, Lithacrosiphon kükenthali Fischer, Thalassema baroni GREEFF und Bonellia thomensis Fischer.

In einer anderen Arbeit über die Sammlung des Reichsmuseums zu Stockholm, meldet FISCHER noch folgende Arten aus dem betreffenden Gebiet: Physcosoma scolops Selenka und de Man gesammelt von C. Bovallius, Phascolosoma pellucidum Keferstein, Dendrostoma alutaceum Grube, Aspidosiphon mülleri Diesing und Lithacrosiphon odhneri Fischer, alle gesammelt von A. v. GoËs.

Unter den von. Dr. VAN DER HORST gesammelten Gephyreën waren 20 Arten, 18 Sipunculiden und 2 Echiuriden, darunter 11 neue Arten.

Von Sipunculiden fanden sich:

Sipunculus nudus Linnaeus, S. robustus Keferstein, Phascolosoma pellucidum Keferstein, Ph. catharinae Fr. Müller, Ph. sluiteri n. sp., Physcosoma varians Keferstein, Ph. scolops Selenka und de Man, $P h$. nigrescens Keferstein, $P h$. horstı n. sp., Ph. microdentigerum n. sp., Ph. minutum n. sp., $P h$. corallicola n. sp., Lithacrosiphon alticonum n. sp., L. poritidis n. sp., Aspidosiphon steenstrupi Diesing, A. fischeri n. sp., A. semperi n. sp., A. gerouldi n. sp.

Von Echiuriden fanden sich: Thalassema baroni Greeff, und Th. kefersteini n. sp.

Die von KÜkenthal und HartmeYer auf Barbados Riff und St. Thomas gesammelte Physcosoma antillarum, Ph. dentigerum, Ph. pectinatum, Aspidosiphon speciosus, A. spinoso-scutatus, Lithacrosiphon kükenthali und Siphonosoma occidentale und die von A. v. GoËs auf St. Barthélemy gesammelte Dendrostoma alutaceum, Aspidosiphon mülleri und Lithacrosiphon odhneri waren in dieser Sammlung nicht vorhanden, indem Sipunculus nudus, S. robustus, Phascolosoma catharinae und die von mir neu beschriebene Arten sich nicht fanden in ihrer Sammlung.

Vielleicht kann man die Tatsache, dass sich unter VAN DER HORST's Material so viele neue Arten befanden, hierdurch erklären, dass er hauptsächlich in Korallen lebende Tiere gesammelt hat.

Hier folgt eine Beschreibung der verschiedenen Arten. 


\section{SIPUNCULOIDEA.}

Von der Gattung Sipunculus waren zwei Arten vorhanden: Sipunculus nudus L. und Sipunculus robustus Keferstein.

\section{Sipunculus nudùs L.}

Spaansche Water, im Sande 4. V. '20, 4 Ex.

Der Körper zeigt 30-33, meistens 32 Längsmuskeln; Anastomosen kommen vor. Es sind 4 Retraktoren vorhanden, die in gleicher Höhe entspringen, die ventralen vom 2.-6. Längsbündel, die dorsalen vom 7.-11.. Sie vereinigen sich nicht bald, erst dicht unterhalb der Tentakel.

Der Darm bildet nur wenige Windungen und ist durch eine Menge Befestiger mit der Körperwand verbunden. Ein Spindelmuskel ist vorhanden; er tritt am Ende der Spira aber nicht heraus. Der Enddarm ist ziemlich lang und bildet ein Divertikel. Der After ist ein querer, von radiären Furchen umstellter Spalt.

Es sind zwei kontraktile Gefässe am Ösophagus vorhanden. Die Segmentalorgane erstrecken sich bis zur Basis der Retraktoren und sind an dem ersten Viertel oder Fünftel ihrer Länge befestigt. Die Segmentalöffnungen liegen 7 Ringmuskelbreiten vor dem After.

Der Rüssel ist bis auf einen schmalen glatten Ring hinter den Tentakeln dicht mit Papillen besetzt. Seine Grösse beträgt etwa ein Viertel der Körperlänge. Nach GruBE sollen die Tiere eine Länge von 2 Fuss erreichen können. Die Exemplare, welche ich untersuchte, massen ohne Rüssel ungefähr $40 \mathrm{~mm}$. Haken sind nicht vorhanden. Am Rektum befinden sich die büschelförmigen Körper, drüsenartige Gebilde.

Sipunculus nudus L. ist bekannt von folgenden Fundorten: Mittelmeer; Nordsee (FoRBES, MAITLAND); Zamboango, Uhoy (SEmPER); Westindien (Würzburger zool. Museum); Florida (Agassiz); Paigntown, Devonshire (Brit. Museum); Malacca (Selenka 1883); Panama (Keferstein 1866); Loyalty Islands (SHIPLEY 1899).

S. nudus ist also eine kosmopolitische Art.

\section{Sipunculus robustus Keferstein.}

Spaansche Water, 18. V.'20, 2 Ex.

Diese zwei Exemplare waren nur klein, das grösste mass etwa $7 \mathrm{~cm}$. ohne den Rüssel, der eine Länge von $2 \mathrm{~cm}$. besass. Die ganze Körperhaut ist durch tiefe parallele Längsfurchen sehr deutlich gerippt. Einige dieser Rippen gehen nicht ganz bis nach vorn, sondern erreichen plötzlich ihr Ende, wodurch die Zahl der Längswülste am Vorderende eine geringere ist als am Hinterende; ich zählte dort 27, hier 30. Der Rüssel ist, wie bei S. nudus L., bis auf einen vorderen glatten Ring, dicht mit Papillen besetzt.

Die 4 Retraktoren entspringen in gleicher Höhe, die ventralen vom 3. -5. Längsmuskel, die dorsalen vom 10.-12.. Die Retraktoren verlaufen, ohne sich zu vereinigen, bis zum Vorderteil des Rüssels.

Der Darm geht eine Strecke nach hinten, steigt wieder nach vorn, kehrt nochmals zurück bis zum Körperende und dann wieder nach vorn zur Afteröffnung. Der After ist ein querer Spalt. Eine Eigentümlichkeit ist, dass vor dem After sich immer zwei Längsrippen befinden, während hinter ihm nur eine Rippe liegt. Der Spindelmuskel ist am Ende nicht befestigt. Es sind zwei kontraktile Schläuche vorhanden.

Die Segmentalorgane sind ziemlich lang; sie erstrecken sich noch über die Basis der Retraktoren hinaus. Ihre Ausmündungsstellen befinden sich 8-9 Ringmuskelbreiten vor dem After. Sie liegen ventral auf je einer Rippe und lassen 7 Längswülste zwischen sich, während der Anus dorsal liegt. Die Segmentalorgane sind nicht durch Mesenterien angeheftet.

Büschelförmige Körper sind nicht vorhanden.

Fundorte von S. robustus Keferstein sind: Uwea, Barbados (Hamburger Museum); Palaos (SEMPER); Amboina (v. MARTEns); Timor (Berliner Museum); Lyly-Inseln (Berliner Museum); Curaçao; Singapore (W. F. LANCHESTER).

Diese Art ist im indo-pazifischen Gebiet häufig, im atlantischen seltener. 
Von der Gattung Phascolosoma waren drei Arten vorhanden: Phascolosoma pellucidum Keferstein, in sehr vielen Exemplaren, Phascolosoma catharinae Fr. Müller und eine neue Art, die ich Phascolosoma sluiteri genannt habe.

Phascolosoma pellucidum Keferstein. Phascolosoma Riisei, Keferstein.

Sipunculus pellucidus Quatrefages.

Phascolosomum pellucidum Quatrefages.

Spaansche Water: 3. IV. '20, 13 Ex.; aus Porites furcata, 13. IV. 20, 2 Ex.; aus Porites furcata, 19. IV. 20, 1 Ex.; 26. IV.'20, 1 Ex.; 25. V. '20, 20 Ex.

Nach SelenkA's Angaben soll der einzige Unterschied zwischen $P h$. Riisei Keferstein und $P h$. pellucidum Keferstein, nämlich der vollständige Mangel der Haken bei den ersten, kein Merkmal sein, das zur Begründung einer neuen Art ausreicht. Unter den Exemplaren, die ich untersucht habe, waren auch zwei, denen die Haken ganz fehlten, und auch sonst stellte ich eine wechselnde Anzahl bei den verschiedenen Individuen fest. W. F. LANCHESTER gibt an, dass unter den von ihm bei Singapore gefundenen Exemplaren keines Haken besass.

Beschreibung: Der Körper ist ziemlich schlank, 5-10 mal so lang als dick, der Rüssel misst. etwa $\frac{1}{2}-\frac{1}{3}$ der Körperlänge. Körper und Rüssel sind blassrötlich, etwas durchscheinend und irisierend. Der Körper ist mit sehr vielen kleinen Papillen bedeckt, an der hinteren Körperspitze stehen dieselben dichter gedrängt und sind öfters stachelförmig ausgezogen, auch an der Rüsselbasis sind sie grösser und zahlreicher als in der Körpermitte.

Die Tentakel stehen in einem doppelten Kranz, der innere aus 8, der äussere aus etwa 14 Tentakeln bestehend.

Die Längsmuskelschicht ist kontinuierlich, nicht in Stränge gesondert. Die 2 Retraktoren entspringen im mittleren Körperdrittel, sie sind ziemlich dünn und vereinigen sich erst ganz vorn. Es ist ein kontraktiler Schlauch vorhanden. Die zwei kleinen Segmentalorgane sind ganz frei. Nach den Angaben SELENKA's sollen die Segmentalorgane von halber Körperlänge sein, während KefERSTEIN angibt: Segmentalorgane kurz. Diese Exemplare stimmen mehr überein mit der Beschreibung KefERSTEIN's, da die Segmentalorgane nicht mehr als ein Viertel oder ein Drittel der Körperlänge erreichen.

Der Darm hat etwa 20 Doppelwindungen, die hinten ganz frei in der Leibeshöhle liegen. Der Enddarm mündet hinter den Segmentalorganen.

Unter den am 3. IV. 20 gesammelten Exemplaren war eins, das nur ein einziges Segmentalorgan besass. Da alle übrigen Exemplare deren zwei haben, vermute ich, es hier mit etwas Zufälligem zu tun zu haben.

Die am 13. IV. 20 gesammelten Exemplare sind dunkler als die übrigen. Ihre Farbe in konserviertem Zustande war nicht blassrötlich, sondern grau. Auch fehlten ihnen die Haken ganz. Da sie aber in allen andern Merkmalen mit Ph. pellucidum Kef. übereinstimmen, meine ich sie doch zu dieser Art rechnen zu müssen.

Ph. pellucidum Kef. ist bekannt von den folgenden Fundorten: St. Thomas, Antillen, (KeFERStein); Rio de Janeiro (Selenka); Bohol, Mindanao, Philippinen, (Semper); Singapore (v. Martens); Torresstrait (Britisches Museum); Malakka (LANCHESTER).

Er ist weit verbreitet im atlantischen und indo-pazifischen Ozean.

Phascolosoma catharinae Fritz Müller. Spaansche Water, aus Porites furcata, 13. IV.'20, 2 Ex.

Farbe in konserviertem Zustande grauweiss. Der Körper ist durchscheinend und bedeckt mit Papillen, die am Hinterende des Körpers und an der Rüsselbasis etwas grösser und zahlreicher sind. In der Mitte des Körpers hatten sie eine Höhe von 0,044 mm, am Hinterende eine Höhe von 0,060$0,068 \mathrm{~mm}$ gegen eine mittlere Dicke von $0,040 \mathrm{~mm}$, und an der Rüsselbasis eine Höhe von 0,072$0,080 \mathrm{~mm}$ und eine mittlere Dicke von $0,028 \mathrm{~mm}$. SELENKA gibt in seiner Beschreibung etwas grössere Zahlen, aber er gibt eine Körperlänge von $40 \mathrm{~mm}$ an, während diese Exemplare nur $10 \mathrm{~mm}$ massen.

Der Körper ist etwa sechsmal so lang als dick. Der Rüssel ist dünner als der Körper und etwas 
länger. Keine Haken. Zahlreiche fadenförmige Tentakel. Zwei lange; dünne Retraktoren am Ende des mittleren Körperdrittels nahe bei einander entspringend. Sie vereinigen sich erst ganz vorn am Rüssel.

Ein mit kurzen Zotten versehener kontraktiler Schlauch begleitet den Ösophagus.

Der Darm bildet ca. 16 Windungen und ist hinten nicht durch den Spindelmuskel befestigt. Der Enddarm ist kurz und mündet hinter den Segmentalorganen.

Die zwei Segmentalorgane sind kurz, von $\frac{1}{2}-\frac{1}{3}$ Körperlänge. Sie sind nicht durch Mesenterien angeheftet.

Die Längsmuskulatur ist nicht in Stränge gesondert.

Diese Art ist bekannt von Desterro, Brasilien (FriTz Mứller) und von Callao (SANDER).

Phascolosoma sluiteri nov. spec. (Fig. 1).

Spaansche Water, aus Korallen, 12 Ex.

Die grösste Merkwürdigkeit dieser Art ist, dass erstens keine Tentakel vorhanden sind, so dass ich auch in Schnittserien keine Spur von Tentakeln entdecken konnte. Zweitens kommen auf der Mitte des Rüssels lange Papillen vor, regelmässig angeordnet in Längsreihen, drei oder vier hinter einander. Da diese Eigentümlichkeiten von keinen der bis jetzt bekannten Tiere beschrieben sind, habe ich gemeint, sie als eine neue Art, Phascolosoma sluiteri, beschreiben zu müssen.

Die Tiere sind sehr schlank, ihr Körper ist 10 bis 12 mal so lang als dick. Das grösste Exemplar mass in konserviertem Zustand ohne Rüssel $13 \mathrm{~mm}$. Der Rüssel ist ungefähr von Körperlänge.

Die Längsmuskulatur ist kontinuierlich, nicht in Stränge gesondert.

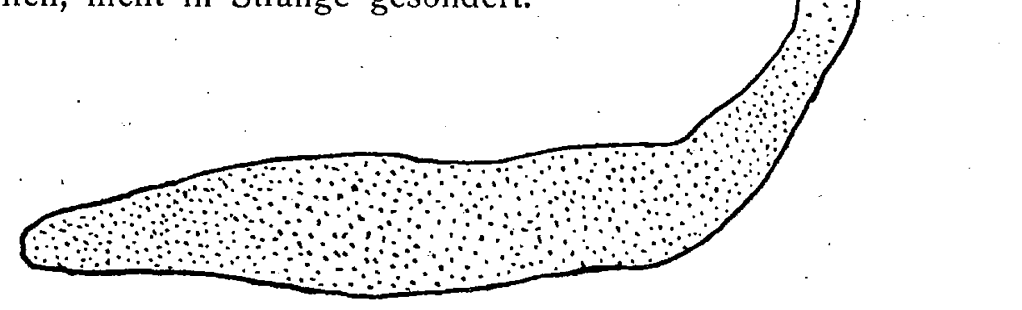

Fig. 1:

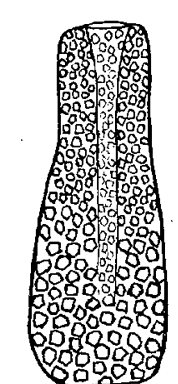

Fig. 2. Papille am Hinterende des Körpers von Ph. sluiteri.

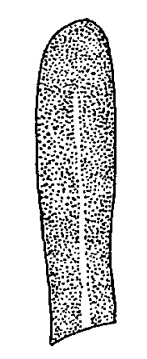

Fig. 3. Papille am Rüssel von $P h$. sluiteri.

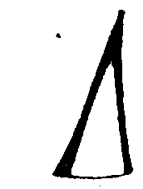

Fig. 4. Haken von $P h$. sluiteri.
Zwei lange dünne Retraktoren entspringen im hinteren Körperdrittel und vereinigen sich erst ganz vorn am Rüssel. Ein kontraktiler Schlauch ist vorhanden, er ist ganz einfach.

Der Darm hat etwa 28 Windungen. Es ist ein Spindelmuskel vorhanden, der aber am Ende der Spira nicht heraustritt. Vorn 3 Befestiger des Darmes an den ersten Windungen. Der Enddarm ist lang und mündet vor den Segmentalorganen. Die zwei Segmentalorgane `sind kurz, von $\frac{1}{4}$ Körperlänge und ganz frei.

Der ganze Körper ist mit vorragenden Papillen bedeckt, die am Hinterende des Körpers am grössten sind. (Fig. 2).

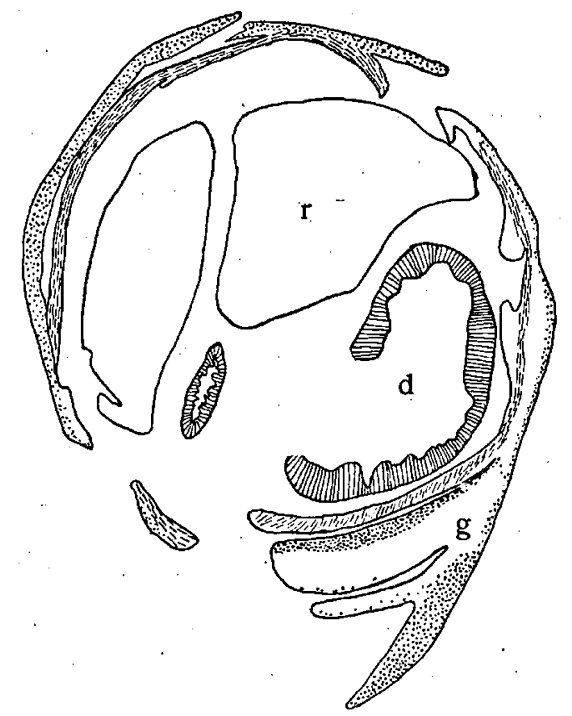

Fig. 5. Querschnitt durch Ph. sluiteri auf der Höhe des Ganglions. $\mathrm{r}=$ Retractor. $\mathrm{d}=$ Darm.

Dort erreichen sie eine Höhe von $0,10 \mathrm{~mm}$ bei einer mittleren Dicke von 0,044 $\mathrm{mm}$, weiter nach vorn zu werden sie allmählich kürzer und dicker. In der Mitte des Körpers sind sie 0,064 mm 
hoch und auch ca. 0,064 mm dick. Die oben genannten 'Papillen am Rüssel erreichen (Fig. 3) eine Länge von $1 \mathrm{~mm}$ und eine mittlere Dicke von $0,20 \mathrm{~mm}$.

Haken sind nur bei einigen kleineren Exemplaren vorhanden (Fig. 4) und sind dann sehr einfach, $0,060 \mathrm{~mm}$ hoch und nur $0,020 \mathrm{~mm}$ breit. Da sie bei den grösseren Exemplaren fehlen, meine ich, dass sie beim Älterwerden ausfallen.

Physcosoma war in 7 Arten vorhanden:

Physcosoma varians Keferstein, Physcosoma scolops Selenka \& De Man, Physcosoma nigrescens Keferstein und 4 neue Arten, die ich genannt habe: Physcosoma horsti, Physcosoma minutum, Physcosoma microdentigerum und Physcosoma corallicola.

Merkwürdig ist, dass fast alle Physcosomen gesammelt sind in der Caracas-Bai, ausgenommen einige Exemplare von Ph. nigrescens Keferstein, und alle Phascolosomen im „Spaansche Water.”

Die Arten $P h$. antillarum Keferstein, $P h$. dentigerum Selenka und de Man und $P h$. pectinatum Keferstein, die sonst von diesen Gegenden bekannt sind, waren unter den von Dr. vAN DER HORST gesammelten Tieren nicht vorhanden.

Physcosoma varians Keferstein.

Phascolosoma Puntarenae Keferstein.

Phascolosoma varians Quatrefages.

Phascolosoma varians Keferstein.

Phymosoma varians Keferstein.

Phascolosoma perlucens. Baird.

Caracas-Bai, aus Algen, 3. V. '20, 2 Ex.; 5. V.'20, 1 Ex.; aus Korallen, 5. V.'20, 1 Ex.

Wir folgen hier grössenteils der Beschreibung KEFERSTEIN's:

Der Körper ist drei bis viermal so lang als dick; der Rüssel ist länger als der Körper. Die Haut ist dünn, etwas irisierend, mit vielen zerstreuten, grossen, kuppelförmigen, abgerundeten Papillen, die an der Rückenseite des Tieres dunkel pigmentiert sind. Sie sind braun mit einem hellen Zentrum und gruppieren sich oft zusammen zu marmoriert aussehenden Flecken. Sie haben einen Durchmesser von $0,20-0,22 \mathrm{~mm}$. Die obere Hälfte des Rüssels hat nur kleine Papillen, ist aber dunkelbraun, mit Querbinden pigmentiert. Der Rüssel trägt zahlreiche, dichtstehende Hakenreihen. Die Haken sind sehr charakteristisch, bilden sehr breite Blätter mit oberer, rechtwinklig umgebogener Spitze und mit grosser abgerundeter Nebenzacke am inneren Rande. Sie sind 0,080 mm hoch und 0,096 mm breit, also etwas grösser als SELENKA angibt. Zwischen den Hakenreihen ziemlich regelmässig angeordnete Hakenpapillen. Vorn am Rüssel, hinter den Tentakeln, eine ganz glatte Zone. Die 20 bis 28 kurzen Tentakeln stehen an der dorsalen Seite des Mundes in zwei seitlichen Reihen.

Die Längsmuskeln sind in etwa dreissig, vielfach anastomosierende Längstränge gesondert.

Vier Retraktoren, von denen die ventralen sich hinten, die dorsalen vorn im mittleren Körperdrittel ansetzen. Die gleichseitigen vereinigen sich erst in der vorderen Hälfte des Rüssels. Zwei grosse Augenflecken.

Der Darm hat nur wenig Windungen, 6 bis 8, mit vollständigem Spindelmuskel, der hinten im Körper inseriert. Unten an der Speiseröhre und an der ersten Windung ein Befestiger. Der Enddarm ist ziemlich lang. Der kontraktile Schlauch ist einfach. Die Segmentalorgane sind lang, im vorderen Drittel durch ein Mesenterium befestigt.

Dem am fünften Mai gesammelten Exemplar fehlte ein Retraktor. Unter den Exemplaren von 3. V. 20 war eins, das eine dicke Haut hatte und viele, sehr grosse und dicht gedrängt stehende $\mathrm{Pa}-$ pillen, also übereinkam mit der zweiten Varietät, die SElenka beschrieben hat von West-Indien.

Fundorte von Ph. varians Keferstein sind: St. Thomas, Vicques (Keferstein); St. Croix (Grube); St. Jan (Grube); Barbados (Wessel); Antillen (Grube); Florida (Agassiz); Bahamas (Shipley); Bermudas (E. L. MARK); Ascension Island (W. H. Brown); Rotuma; Funafuti.

Diese Art ist also häufig im atlantischen Ozean. und tritt auch im pazifischen auf. 
Physcosoma scolops Selenka und De Man.

Caracas-Bai, 19. IV. '20, 1 Ex.

Dieses Tier ist etwas kleiner als das von SelEnKa und DE MAN beschriebene und auch sind die Haken nur $0,056 \mathrm{~mm}$ hoch und breit, statt $0,070 \mathrm{~mm}$ hoch und $0,074 \mathrm{~mm}$ breit.

Die dunklen Linien, die den mittleren hellen Raum der Haken begrenzen, sind sanft gebogen, nicht geknickt. Da das Tier übrigens mit den hier folgenden Beschreibungen SELENKA's übereinstimmt, glaube ich es doch zu Ph. scolops Sel. und De Man rechnen zu können.

Körper von schlanker Gestalt, 6-9 mal so lang als dick; Rüssel fast so lang wie der Körper. Die Längsmuskeln schimmern durch die Haut hindurch.

Nach SEMPER's Angaben über lebende Tiere sollen die.Weibchen rötlich, die Männchen dunkelbläulich erscheinen, so dass man schon auf den ersten Blick die beiden Geschlechter unterscheiden kann. Die gleiche Beobachtung hat SElenka an lebenden Exemplaren der Ph. granulatum gemacht. Der Rüssel ist graulich und zeigt auf seiner Rückenseite meistens mehrere unregelmässige rothbraune oder gelblichrote Halbbinden oder Flecken.

Der ganze Körper ist mit unregelmässig zerstreuten, ungleichen, kleinen, kegelförmigen Papillen bedeckt; dieselben heben sich an der vorderen Körperhälfte kaum durch ihre dunkle Farbe von der hellgefärbten Haut $a b$; sie sind oval, zeigen an der Spitze einen hellen, die Mündungsöffnung einschliessenden Raum, welcher durch mehrere Reihen unregelmässiger, bräunlicher, nach der Peripherie hin allmählich kleiner werdender Plättchen umgeben wird. Am hinteren Körperende werden die Papillen allmählich bedeutend grösser und fallen dort durch ihre rothbraune Färbung auch dem unbewaffneten Auge auf. Dicht vor dem After an der Rüsselbasis sind sie ebenfalls sehr gross und zahlreich, von rotbrauner Farbe, gerade wie die am hinteren Körperende gelegenen; im hinteren Rüsseldrittel bekommen diese Papillen eine schlankere, spitz kegelförmige Gestalt, die dorsalen an Grösse die ventralen übertreffend. Gegen die Tentakel hin werden die Rüsselpapillen allmählich kleiner und niedriger und hören in einiger Entfernung hinter den letzten Hakenringen auf, so dass dieser Rüsselteil glatt erscheint. Es sind nicht viele Hakenreihen vorhanden, 15 bis 17. Zwischen den Hakenringen liegen kreisrunde, sehr kleine Hakenpapillen.

Die Zahl der Tentakel ist 12, sie stehen in einem an der Rückenseite unterbrochenen Kreise, oberhalb der Mundöffnung, und sind innen braun gefärbt.

Die Muskelschicht zeigt 20 bis 22 sehr wenig anastomosierende Längsmuskeln, welche durch die Haut hindurchschimmern und dicht vor dem After zu einem kontinuierlichen Schlauche verschmelzen. Die vier Retraktoren entspringen im mittleren Körperdrittel, nicht weit von einander; die breiteren ventralen von 5 Längsmuskeln am hinteren Rande des mittleren Körperdrittels, die schmäleren dorsalen von 3 Längsmuskeln in kurzer Entfernung von den ersteren. Die gleichseitigen Retraktoren gelangen schon hinten im Rüssel zur Vereinigung. Der kontraktile Schlauch ist einfach. Die Darmspira hat nur wenige Windungen, Selenka gibt 12 bis 14 an. Der Enddarm ist ziemlich lang und mündet hinter den Segmentalorganen. Der Spindelmuskel ist vollständig, verlässt hinter die Spira und inseriert im Körperende.

Zwei Segmentalorgane, fast von halber Körperlänge, im letzten Drittel oder Viertel frei.

Diese Art ist bekannt von folgenden Fundorten:

Philippinen (SEmPer); Singapore (v. Martens); Koseir, Rotes Meer (Klunzinger); Durban (Weber); West-Afrika, Goldküste (Dr. Brauns); Isla das Rolas (R. GreefF); Isla Annodon (Arnold Schultze); Mauritius; Sansibar; St. Barthélemy (A. v. Gö̈s); St. Thomas, Westindien (C. Bovallius); Madagaskar; Australien; Tasmanien; Kermadee Islands; Loyalty Islands.

$P h$. scolops ist also eine sehr verbreitete Art, die sowohl im indischen Ozean als auch im pazifischen und im atlantischen vorkommt. Von Westindien war sie schon bekannt. FISCHER will diese Art auffassen als eine Varietät von Ph. granulatum F. S. Leuckart. Diese Art ist aber allein vom Mittelmeer und adriatischen Meer bekannt, während $P h$. scolops sich in ihrer Verbreitung auf die Tropen beschränkt. Ich möchte sie lieber als zwei verschiedene Arten behalten, wie SLUiTER es tut. 
Physcosoma minutum nov. sp.

Curaçao 1 Ex.

Der Körper ist gedrungen, nur zweimal so lang als dick, am Hinterende abgerundet. Der Rüssel ist mehr als zweimal so lang als der Körper.

Die ganze Körperhaut trägt bräunliche Papillen, die am Hinterende des Körpers eine Höhe von $0,16-0,20 \mathrm{~mm}$ und eine Breite von $0,18 \mathrm{~mm}$ erreichen (Fig. 6).

Die Längsmuskelschicht ist in etwa 26 vielfach anastomosierende Längsstränge gesondert.

4 ziemlich kurze Retraktoren, hinten im mittleren Körperdrittel nicht weit von einander entspringend. Die gleichseitigen Retraktoren vereinigen sich unmittelbar. Die breiteren ventralen entspringen von 7 Längsmuskeln, die dünneren dorsalen in geringer Entfernung von den ersteren von 3 bis 4 Längsmuskeln. 20 Tentakel. Sehr

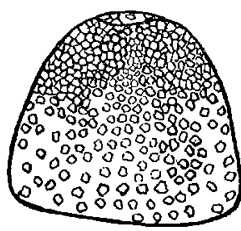
viele Hakenreihen, etwa 100. Die Haken sind ziemlich stark gebogen, spitz zulaufend (Fig. 7), mit einer Nebenzacke am inneren Rande. Vereinzelt kommen auf dem Körper noch einige vor. Sie sind $0,052-0,060 \mathrm{~mm}$ hoch und $0,068 \mathrm{~mm}$ breit. Charakteristisch ist der Verlauf der dunklen Linien um den hellen Raum der Haken.

Der Darm bildet nur wenige Windungen (8). Ein vollständiger Spindelmuskel, der hinten im Körper inseriert, ist vorhanden. Der Enddarm ist ziemlich lang und ist mit Mesenterien am Ende festgeheftet; er mündet hinter den Segmentalorganen. Die żwei Segmentalorgane sind lang, fast von Körperlänge und ganz frei.

Die Ösophagus ist begleitet von einem kontraktilen Schlauch, der mit kleinen Zotten versehen ist.

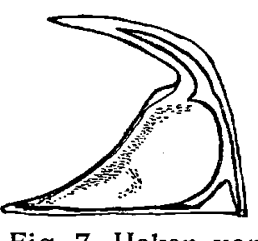

Fig. 7. Haken von Ph. minutum.

Der Körper ist ohne den Rüssel nur $5 \mathrm{~mm}$ lang.

Physcosoma nigrescens (Keferstein).

Phascolosoma nigrescens Keferstein.

Sipunculus nigrescens Quatrefages.

Phymosomum nigrescens Quatrefages.

Phascolosoma nigrescens Baird.

Phascolosoma planispinosum Baird.

Phymosoma nigrescens Keferstein.

Caracas-Bai, 7.IV.'20, 1 Ex.; 5. V. '20, 1 Ex.; 6. V. '20, 2 Ex.; 25. V. '20, 1 Ex.

Spaansche Water, aus Korallen, 5. V.'20, 1 Ex.

Spaansche Haven, 20. V.'20, 4 Ex.

Spaansche Water, 25. V.'20, 1 Ex.

Der Körper ist ziemlich gedrungen, vier- oder fünfmal so lang als dick. Der Rüssel ist dünner und ein wenig länger als der Körper. Die vier Retraktoren entspringen hinten im mittleren Körperdrittel, nicht in gleicher Höhe; die gleichseitigen vereinigen sich bald. Die breiteren, ventralen Retraktoren entspringen von 6 Längsmuskeln, die schmäleren dorsalen, in einiger Entfernung von diesen, von 3 Längsmuskeln.

Die Längsmuskelschicht ist in etwa dreissig, vielfach anastomosierende Längsstränge gesondert.

Der Darm hat nur wenig Windungen, etwa 14. Der Enddarm ist meistens ziemlich kurz, aber variiert bei den verschiedenen Exemplaren. Die Mündungsöffnung des Enddarms liegt hinter denen der Segmentalorgane.

Ein vollständiger Spindelmuskel, welcher sich am hinteren Körpérende inseriert ist vorhanden.

Der kontraktile Schlauch hat viele kleine Zotten.

Die Zahl der Tentakel beläuft sich auf etwa 20. Sie sind in einem, in der.Rückenlinie unterbrochenen Kranze angeordnet. Dicht hinter den Tentakeln liegen die geschlossenen Hakenreihen, deren Zahl meistens sehr bedeutend ist. Die Haken haben eine scharfe, stark gekrümmte Spitze; ihr innerer Rand trägt eine grosse Nebenspitze. Bei den von mir untersuchten Exemplaren variierte ihre Höhe von $0,052-0,072 \mathrm{~mm}$, ihre Breite von 0,058-0,080 mm. Selenka gibt eine Höhe von 0,09 $\mathrm{mm}$ und eine Breite von 0,10 $\mathrm{mm}$ an und KefERSTEIN eine Höhe und Breite beide von 0,084 mm.

Die beiden Segmentalorgane sind kurz, kaum von halber Körperlänge, das hintere Viertel, Drittel 
oder die hintere Hälfte nicht durch Mesenterien angeheftet. Der ganze Körper, sowie der Rüssel, ist mit ziemlich stark hervorragenden kegelförmigen Papillen bedeckt, die besonders am Hinterende des Körpers und an der Rüsselbasis sehr stark hervorragen und auch dichter gedrängt stehen. Die Papillen sind dunkler pigmentiert als die Haut. An der Spitze liegt inmitten eines hellen Hofes die feine Mündungsöffnung. Der helle Hof war bei den von mir untersuchten Tieren von sehr kleinen Körnern umgeben, wie SelenKa es angibt für die Exemplare der Fidji-Inseln, und nicht von mehreren Reihen ziemlich grosser, unregelmässig gestalteter Plättchen, wie er es fand bei den Philippinischen und bei den von Mauritius stammenden Tieren. Am Hinterende des Körpers erreichen die Papillen eine Höhe von $0,12-0,14 \mathrm{~mm}$ und eine Breite von $0,15 \mathrm{~mm}$.

Auch die zwischen den Haken liegenden Rüsselpapillen zeigen die Gestalt, welche SelENKA beschreibt für die Exemplare von den Fidji-Inseln, nämlich die obere Hälfte der Papille ist umlagert von kleinen unregelmässigen Körnchen.

Die Tiere leben nach SEMPER in Korallenbänken und sind sehr kontraktionsfähig.

$P h$. nigrescens ist die einzige Art von Physcosoma, von welcher auch Exemplare, zwar nur zwei, im Spaansche Water gefunden wurden, während sonst alle Physcosomen in der Caracas-Bai gesammelt sind.

Es besteht ein grosser Unterschied zwischen Caracas-Bai und Spaansche Water, wie VAN DER HoRst es angibt in seinem Artikel: Narrative of the Voyage and short description of localities. Er sagt dort:

"There is a great difference between the Caracas Bay and the Spanish Water, though they are separated from each other by a narrow neck of land, at some places measuring across only some tens of metres. The Spanish Water is like an inland sea, remainder as it is of the lagoon, dating from the long past times when Curaçao was an atoll. It has many bays and some small islands and stands only in communication with the Caribbean Sea by the rather narrow Spanish Port. As the tides are of small importance, the renewal of the water is not great, though it is sufficient to prevent any considerable in- or decrease in the salinity during dry or rany times resp. Compared with the clear blue ocean water of Caracas Bay, the Spanish Water is muddy, I could not look deeper than two metres here. The bottom too consists largely of mud mixed with stones. As a matter of course this difference has a great influence on the animal life."

Er fand schon Unterschiede zwischen den Arten von Korallen und Spongien, die er in der CaracasBai und Spaansche Water gesammelt hat, und dasselbe kann ich also auch von den Gephyreen sagen.

Fundorte von $P h$. nigrescens sind:

Fidji-Inseln (Keferstein); Philippinen: Uhoy, Luzon, Mindanao, Camiguin (SemPer und F. Jager); Mauritius (MöBIUS); Koseir, Rotes Meer (KLunzinger); Kapverdesche Inseln (R. PAEssLer); Goldküste (C. Hupfer); Isla Annobon (Arnold Schultze); Honolulu (Eugenie Exp.); Rotuma und Funafuti; Loyalty Islands; St. Thomas; St. Jan.

Die Art hat zirkumtropische Verbreitung; sie tritt am häufigsten im pazifischen und indischen Ozean auf; ist weniger verbreitet im atlantischen.

Physcosoma microdentigerum nov. sp.

Caracas-Bai : aus Maeandrina, 7.IV.'20, 10 Ex.; in Stein, 7. IV.'20, 1 Ex.; 7. IV. '20, 2 Ex.; 19. IV. '20, 1 Ex.; zwischen Steinen, 11. V.'20, 1 Ex.; aus Korallen, 13. V.'20, 5 Ex.; 25. V. '20, 1 Ex.

Der Körper ist 4 bis 6 mal so lang als dick, der Rüssel von Körperlänge. Die Körperlänge beträgt ohne den Rüssel etwa $10 \mathrm{~mm}$. Die Körperhaut ist durchscheinend. Am Hinterende ist der Körper zugespitzt.

Der ganze Körper ist mit Papillen bedeckt; in der Mitte sind diese Papillen wenig zahlreich, sehr klein und flach, an der Rüsselbasis und am Hinterende des Körpers sind sie gross, braun gefärbt und in grosser Anzahl vorhanden.

Die Papillen sind kegelförmig mit ovaler Grundfläche und hellem, von oben gesehen kreisförmigem Gipfel, welcher die kleine zentrale Öffnung trägt. Rings um die Öffnung liegen konzentrisch angeordnete kleine Plättchen und an der Peripherie etwas kleinere Plättchen. (Fig. 8).

Am Hinterende des Körpers erreichen sie eine Höhe von 0,14 mm und haben einen Durchmesser von $0,10 \mathrm{~mm}$. Dorsal an der Rüsselbasis sind einige der Papillen zu grossen nach hinten 
gerichteten Stacheln ausgezogen (Fig. 9). Diese werden aber bei weitem nicht so gross wie die Dornen von Ph. dentigerum Selenka und De Man aus dem indo-pazifischen Gebiet. Sie erreichen eine Länge von $0,16-0,25 \mathrm{~mm}$, wovon der Stachel fast die Hälfte einnimmt. Nach der ventralen Seite nehmen die Papillen allmählich an Grösse ab und auch nach den Tentakeln zu werden sie regelmässig kleiner.

Vorn am Rüssel liegen die Hakenreihen, die nicht in sehr grosser Anzahl vorhanden sind. Ihre Zahl variierte von 15 bis 40 . Die Haken sind ziemlich breit, haben eine scharf gekrümmte Spitze und eine grosse Nebenspitze am inneren Rande (Fig. 10). Sie sind bis $0,060 \mathrm{~mm}$ hoch und unten 0,064 bis $0,068 \mathrm{~mm}$ breit.

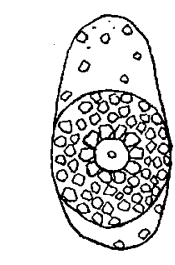

Fig. 8. Papillen am Hinterende des Körpers von Ph. micro-
dentigerum.

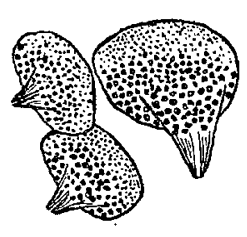

Fig. 9. Stacheln von Ph. microden- Fig. 10. Haken von tigerum.

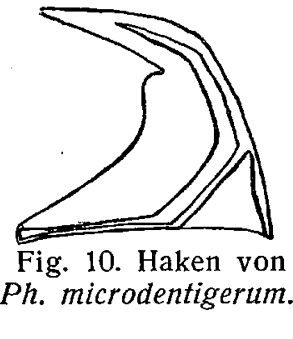

Die 4 Retraktoren entspringen nicht in gleicher Höhe, die breiteren ventralen entspringen von 5-7 Längsmuskelsträngen, die schmäleren dorsalen in geringer Entfernung von diesen von 2, 3 oder 4 Längsmuskeln.

Die gleichseitigen Retraktoren vereinigen sich bald.

Die Darmspira hat etwa 10 Windungen und einen vollständigen Spindelmuskel, der in der hinteren Körperspitze inseriert. Der Enddarm ist lang und mündet etwas hinter den Segmentalorganen. Der Ösophagus ist begleitet von einem mit kleinen Zotten versehenen kontraktilen Schlauche.

Die Segmentalorgane sind kurz, von halber Körperlänge; nur ihr hinteres Drittel ist nicht mit Mesenterien festgeheftet.

10 Tentakel, innen schwarz gefärbt, von einem Wall umgeben. Die Längsmuskulatur ist in 20 bis 30 öfters anastomosierende Stränge gesondert.

Ich habe diese Art, die in vielen Hinsichten übereinstimmt mit $P h$. dentigerum, aber von ihr abweicht durch die geringere Grösse der Stacheln, durch den mit kleinen Zotten versehenen kontraktilen Schlauch und einige andere untergeordnete Merkmale, Ph. microdentigerum genannt.

\section{Physcosoma horsti nov. sp.}

Caracas-Bai, 25. V. '20, 5 Ex.

Die Körperhaut dieser Tiere ist sehr stark irisierend. Die Muskeln hatten sich bei den konservierten Tieren von der Körperhaut losgelöst. Der Körper ist braun marmoriert, 5 bis 6 mal so lang als dick, der Rüssel von Körperlänge. Ohne den Rüssel ist der Körper etwa $10 \mathrm{~mm}$ lang. Der ganze Körper ist mit ziemlich grossen Papillen bedeckt, die am Hinterende des Körpers und an der Rüsselbasis nicht viel grösser sind als in der Mitte des Körpers. Die ganze Papille ist bedeckt mit kleinen Körnchen, in der Mitte findet man eine kleine Ausmündungsöffnung. Sie erreichen eine Höhe bis 0,2 mm.

Vier breite, kurze Retraktoren, die dicht neben einander entspringen, die dorsalen von fünf Längsmuskeln, die breiteren ventralen von acht. Die gleichseitigen vereinigen sich bald.

Die Längsmuskulatur ist in ungefähr dreissig, sehr stark anastomosierende Stränge gesondert.

Der Darm hat nur acht Windungen, mit vollständigem Spindelmuskel, der sich hinten im Körperende inseriert. Der Enddarm ist lang und mündet in gleicher Höhe mit den Segmentalorganen. Ein kontraktiler, mit kleinen Zotten versehener Schlauch begleitet den Ösophagus.

Die zwei Segmentalorgane sind kurz, von halber Körperlänge, und sind zur Hälfte durch Mesenterien festgeheftet.

Tentakel sind nur 8 vorhanden.

Viele Hakenreihen; die Haken stehen ziemlich weit von einander und sind sehr winzig. Ihre Höhe ist nur $0,028 \mathrm{~mm}$, ihre Breite $0,036 \mathrm{~mm}$. (Fig. 11).

Die Form der Haken und der Verlauf der hellen Linien ist sehr einfach. Zwischen Fig. 11. Haken den Haken stehen die Ausmündungspapillen der Hautkörperchen, die sehr gross sind von Ph. horsti. und mit kleinen Körnchen bedeckt. Sie erreichen eine Höhe von 0,72 mm und einen Durchmesser von $0,60 \mathrm{~mm}$. 
Physcosoma corallicola nov. sp.

Caracas-Bai, aus Korallen, 2. IV.'20, 1 Ex.

Dieses Tier war das einzige Exemplar in dieser Sammlung, das keine Haken besass. Leider war es sehr beschädigt, sodass einige Merkmale nicht untersucht worden konnten.

Hier folgt eine- Beschreibung, soweit ich imstande bin sie zu geben.

Der Körper ist fünfmal so lang als dick, der Rüssel ist von halber Körperlänge.

Die Muskelschicht ist in 20 anastomosierende Längsstränge gesondert. Es finden sich 4 lange Retraktoren, die gleichseitigen vereinigen sich unmittelbar. Die dorsalen Retraḳtoren sind sehr dünn, sodass es scheint, als ob nur 2 vorhanden seien.

Die Darmspira hat viele Windungen, war aber sehr beschädigt, sodass ich die genaue Anzahl der Windungen nicht anzugeben vermag. Auch der Enddarm und der kontraktile Schlauch fehlten. Ein vollständiger Spindelmuskel, der hinten im Körper inserierte, war vorhanden.

Die Segmentalorgane sind lang, fast von Körperlänge; der grösste Teil ist festgeheftet.

12 Tentakel. Hirn mit deutlichen Augenflecken. Keine Haken.

Die Körperpapillen am Rüsselende sind sehr flach, kleine Plättchen um eine zentrale Öffnung. (Fig. 12).

Am Ende des Körpers sind die Papillen nicht scharf abgegrenzt.

$\mathrm{Da}$ dieses Tier mit keiner der bisher bekannten Arten übereinstimmt, meine ich es trotz seiner Unvollständigkeit als eine neue Art beschreiben zu können und habe es Physcosoma corallicola genannt. Von der Gattung Lithacrosiphon, von der bis jetzt nur 6 Arten bekannt sind:
Fig. 12. Kör- Lith. maldivensis Shipley; Lith. kükenthali Fischer; Lith. indicus Fischer; Lith. odhneri perpapille von Fischer; Lith. cristatus Sluiter und Lith: uniscutatus Ikeda, sind in dieser Sammlung Ph. corallicola. wieder zwei neue Arten vorhanden, denen ich den Namen Lith. alticonum und Lith. poritidis gegeben habe.

Von dieser Gattung sagt FISCHER: Sie ist charakterisiert durch einen dem Vorderende des Körpers aufsitzenden, schiefen, vollständigen oder abgestumpften Kalkkegel, der entweder ganz oder teilweise in einer chitinigen, bisweilen gefurchten Hautscheide steckt, eine Bildung, die sie

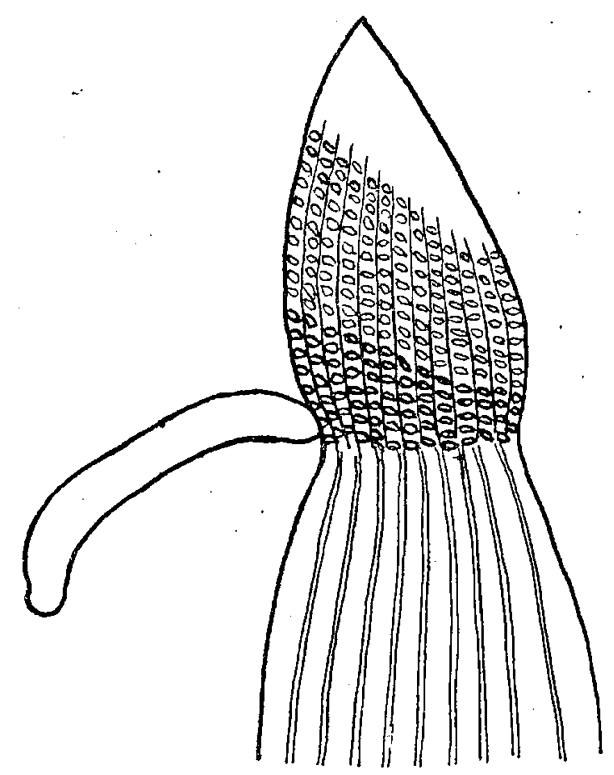

Fig. 13. Kalkkegel von Lith. alticonum wesentlich von der ihr sonst ähnlichen Gattung Aspidosiphon unterscheidet. Hier findet sich am Vorderende des Körpers ein dieses vorzüglich dorsal umgreifendes häufig gefurchtes After- oder Vorderschildchen, das aus einer grossen Anzahl fest aneinander stossender Chitinplatten gebildet ist, bisweilen mit Kalkkörnchen bestreut ist, aber keinerlei Kalkmassen im Innern beherbergt. Ferner besitzt diese Gattung auch am Hinterende eine ähnliche Bildung, die der Gattung Lithacrosiphon fehlt.

Lithacrosiphon alticonum nov. sp.

Caracas-Bai: aus Maeandrina, 7.IV.'20, 2 Ex.; aus Korallen, 13. V.'20, $7 \mathrm{Ex}$.

Der Körper ist irisierend, die Muskeln durch die Haut, besonders im mittleren Teil, durchschimmernd. Der Körper ist etwa viermal so lang als dick, der Rüssel ist von halber Körperlänge. Ohne Rüssel misst das Tier ca. $13 \mathrm{~mm}$.

Am Vorderende des Körpers findet sich ein hoher, konischer, kalkiger Kegel, unten umgeben von einer Hautscheide. Dieser

Kegel ist fast $2 \mathrm{~mm}$ hoch und etwa $1 \mathrm{~mm}$ breit an der Basis. Auf der Hautscheide sind 42 Furchen zu zählen (Fig. 13).

Die Längsmuskulatur ist in etwa 20 gegeneinander stossende, sehr stark anastomosierende, flache Längsstränge gesondert. Ihre Anzahl ist dadurch schwer zu zählen. 
Der Retraktor entspringt weit hinten im Körper, mit 2 getrennten, breiten Wurzeln vom 1. bis 5 . Längsmuskel, die sich bald vereinigen.

Der Ösophagus begleitet den Retraktor nur bis etwa zur Hälfte. Der Darm biegt dann eine Strecke zurück nach vorn, krümmt sich wieder nach hinten und bildet dann etwa 14 Windungen. Der Enddarm ist lang, fast von halber Körperlänge, und hat in der Mitte ein kleines Divertikel und am Ende ein grösseres.

Der After ist ein querer Spalt, er liegt unmittelbar hinter dem Kalkkegelchen und nur etwas vor der Mündung der. Segmentalorgane. Die 2 Segmentalorgane sind sehr lang, länger als der Körper, und sind im Körperende zurückgebogen. Nur das letzte Drittel ist frei.

Die Hakenreihen schimmern durch die Haut des Rüssels hindurch. Die Haken an der vorderen Partie des Rüssels sind einspitzig (Fig. 14), während sie am Hinterende des Rüssels zwei Spitzen tragen (Fig. 15). Die Verdickungsleiste der Haken trägt, wie es auch bei anderen Lithacrosiphonarten wohl der Fall ist,

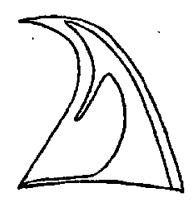

Fig. 14. Einspitziger Haken von Lith. alticonum.

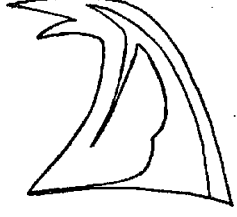

Fig. 15. Zweispitziger Haken von Lith. alticonum.

einen Fortsatz. Die zweispitzigen Haken erreichen eine Höhe von 0,044 mm und eine Breite von $0,040 \mathrm{~mm}$. Die einspitzigen sind etwas kleiner, nämlich $0,032 \mathrm{~mm}$ hoch und $0,034 \mathrm{~mm}$ breit. Zwischen den Hakenreihen stehen die kleinen Mündungsröhrchen der Hautkörperchen, die eine Höhe von 0,013-0,015 $\mathrm{mm}$ erreichen, also ein Drittel der. Hakenhöhe. Stacheln kommen nicht vor. Die Hautkörperchen sind rund; in der Mitte liegt die Mündungsöffnung; rings um die Öffnung liegen konzentrisch angeordnete kleine Plättchen, die nach der Peripherie hin etwas grösser werden.

Am Hinterende des Körpers ist kein Schildchen abgegrenzt, wohl aber sind die Felder ziemlich regelmässig angeordnet, wodurch eine radiäre Furchung entsteht, die etwas braun gefärbt ist.

Diese Art unterscheidet sich also durch die hohe Form des Kalkkegelchens, durch den Besitz ein- und zweispitziger Haken und die zwei Divertikel am Enddarm. Nach der hochkonischen Form des Kegels habe ich sie Lithacrosiphon alticonum genannt.

Lithacrosiphon poritidis nov. spec.

Caracas-Bai, aus Korallen, 2. IV. '20, 1 Ex.

Dieses Tier unterscheidet sich von der vorigen Art durch die etwas geringere Höhe des Kalkkegels, der ebenso hoch wie breit ist; ferner fehlen am Enddarme die Divertikel und die Haken sind alle einspitzig. Auch sind die Segmentalorgane etwas kürzer.

Der Körper ist etwa $4 \mathrm{mal}$ so lang als breit. Der Kalkkegel ist $1 \mathrm{~mm}$ hoch und breit. Der Kegel ist von einer braun gefärbten Hautscheide umgeben. Auf dieser Scheide zählte ich 40 Längsfurchen. Der Rüssel ist kurz. Die Längsmuskulatur ist in dreissig, sehr stark anastomosierende und dadurch schwer zu zählende Stränge gesondert. Zwei Retraktoren, von welchen der eine aus zwei getrennten Wurzeln entspringt, der andere aus einer. Die Vereinigung der Retraktoren findet erst auf der Hälfte ihrer Länge statt.

Der Darm hat etwa zwanzig Windungen, mit vollständigem Spindelmuskel, auch im Enddarm,

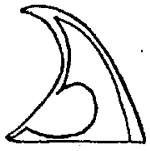

Fig. 16. Haken von Lith. poritidis.

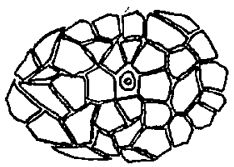

Fig. 17. Körperpapille am Hinterende des Körpers von Lith. poritidis. der hinten im Körper inseriert. Der Enddarm ist lang, von halber Körperlänge, die Mündung befindet sich vor den Segmentalorganen. Ein kontraktiler Schlauch ist vorhanden, er ist ganz einfach.

Die Segmentalorgane sind kaum $\frac{3}{4}$ der Körperlänge, der grösste Teil, etwa $\frac{2}{3}$ ihrer Länge, ist mit Mesenterien angeheftet.

Diese Art trägt vorn 12 Tentakel. Hinter den Tentakeln liegen die Hakenreihen. Die Haken sind alle einfach und haben nur eine Spitze

(Fig. 16). Ihre Höhe beträgt $0,040 \mathrm{~mm}$ und ihre Breite $0,036 \mathrm{~mm}$. Zwischen den Haken stehen dia kleinen Mündungsröhrchen der Hautkörperchen, von etwa ein Drittel der Hakenhöhe. Stacheln fehlen.

An der Rüsselbasis und am Körperende stehen viele kleine Körperpapillen; sie bestehen aus dicht neben einander liegenden Plättchen; die Mündungsöffnung liegt in der Mitte (Fig. 17).

Von Lith. kükenthali Fischer, der auch einspitzige Haken hat, unterscheidet diese Art sich durch 
die Anzahl der Längsmuskelstränge, bei Lith. kükenthali sind nur 18, hier 30 vorhanden, und durch die Anzahl der Längsfurchen der Hautscheide. Lith. kükenthali hat 52 solcher Furchen, Lith. poritidis nur 40. Da das Exemplar, das FISCHER untersucht und beschrieben hat, nur unvollständig war, kann ich betreffs der andern Merkmale keine Vergleichung anstellen; doch scheinen mir diese Unterschiede schon genügend, um dieses Tier von Curaçao als eine neue Art aufzustellen.

Von der Gattung Aspidosiphon sind vier Arten vorhanden: Aspidosiphon steenstrupi und drei neue Arten. Ich habe diese genannt Aspidosiphon semperi, Aspidosiphon fischeri und Aspidosiphon gerouldi.

Aspidosiphon semperi nov. sp.

Caracas-Bai, aus Korallen, 13. V. '20, 7 Ex.

Der Körper ist etwa $15 \mathrm{~mm}$ lang, der Rüssel ungefähr gleich lang. Die Längsmuskulatur ist in 22 bis. 26 anastomosierende Längsbündel gesondert. Es besitzt 4 Retraktoren; die ventralen entspringen vom 3. bis 6. Längsmuskel, die dorsalen vom 6. bis 9. Längsmuskel; die gleichseitigen vereinigen sich bald. Unter den bis jetzt beschriebenen Formen ist keine mit vier Retraktoren, immer waren zwei angegeben und in zwei Fällen, bei $A$. cumingii und A. klunzingeri nur einer.

Die Basis der Retraktoren ist $\frac{1}{4}$ Körperlänge vom hinteren Schildchen entfernt.

Der Darm hat nur sieben Windungen, mit vollständigem, hinten im Körper inserierten Spindelmuskel. Der Enddarm ist lang; die Mündungsöffnung liegt unmittelbar vor denen der Segmentalorgane.'

Die Segmentalorgane sind sehr lang, fast von Körperlänge; sie erstrecken sich bis an die hintere Anheftung der Retraktoren. Das hintere Drittel ist frei, übrigens sind sie durch Mesenterien befestigt.

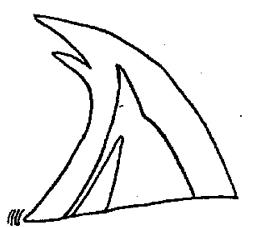

Fig. 18. Haken von A. semperi.

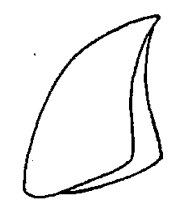

Fig. 19.

Rüsseldorn von A. semperi.

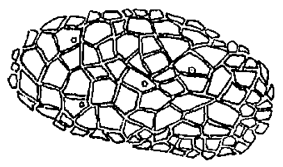

Fig. 20.

Körperpapille von A. semperi.

Acht Tentakel. Hinter diesen die Hakenreihen. Die Haken haben zwei Spitzen und sind $0,054 \mathrm{~mm}$ hoch. (Fig. 18).

Zwischen den Haken die Mündungsröhrchen der Hautkörperchen, die 0,024 bis 0,028 mm hoch sind, also etwa von halber Hakenhöhe. Am hinteren Rüsselende stehen Dornen. Die Dornen sind etwa von Hakenhöhe. (Fig. 19).

Die Hautkörperchen stehen dicht gedrängt, sodass die eckigen Felder gegeneinander stossen. (Fig. 20). Sie sind ungleich gross, in der Mitte des Körpers etwa $0,1 \mathrm{~mm}$ lang, vorn und hinten am Übergang nach den Schildchen mehr als doppelt so gross.

Aspidosiphon fischeri nov. sp.

Caracas-Bai: aus Korallen, 2. IV.'20, 1 Ex.; 1. V. '20., 1 Ex.

Die beiden Tiere sind nur sehr kleine Exemplare. Der Körper misst ohne Rüssel nur $5 \mathrm{~mm}$ und ist viermal so lang als dick.

Die Rüssellänge beträgt fast das Doppelte der Körperlänge.

Die Längsmuskulatur ist in etwa 18 stark anastomosierende Stränge gesondert.

Der Retraktor entspringt unmittelbar vor dem Hinterschildchen mit zwei getrennten Wurzeln, die sich erst in der Mitte der Körperlänge vereinigen. Der Darm hat etwa zwanzig Windungen mit

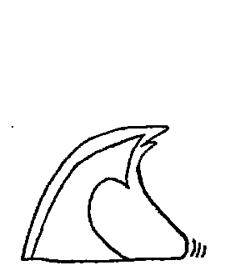

Fig. 21.

Haken von

A. fischeri.

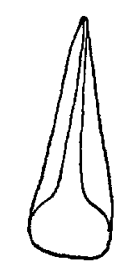

Fig. 22.

Rüsseldorn vo A. fischeri. vollständigem Spindelmuskel, der hinten im Körper inseriert. Der Enddarm ist kurz und mündet hinter den Segmentalorganen.

Die Segmentalorgane sind von halber Körperlänge und etwa zur Hälfte mit Mesenterien festgeheftet.

18 Tentakel. Viele Hakenreihen; die Haken sind klein und haben zwei Spitzen. Sie sind 0,028 $\mathrm{mm}$ hoch und 0,024 $\mathrm{mm}$ breit. (Fig. 21).

Am Hinterende des Rüssels stehen wieder Dornen. (Fig. 22).

Das vordere Schildchen ist flach, nicht gefurcht. Es besteht aus regelmässigen, vieleckigen Felderchen, die in der Mitte kleiner und von dunkelbrauner Farbe, an den Rändern grösser und heller sind. Das hintere Schildchen ist rund, 
nicht scharf abgegrenzt, und hat in der Mitte kleine, dunkle Felderchen, an der Peripherie grössere und hellere, die allmählich in die übrige Körperhaut übergehen.

Der ganze Körper ist bedeckt mit kleinen, wenig hervorragenden Papillen.

Aspidosiphon gerouldi nov. sp.

Port de Paix, Haiti, 6. VI. '20. 1 Ex.

Der Körper ist dreimal so lang als dick, der Rüssel etwas länger als der Körper. Ohne Rüssel beträgt die Körperlänge nur $4 \mathrm{~mm}$.

Die Längsmuskeln bilden zwar keinen kontinuierlichen Schlauch, aber die Anordnung in getrennten Bündeln ist so wenig deutlich ausgeprägt, dass keine gesonderte Bündel zu zählen sind.

Die 2 Retraktoren entspringen unmittelbar vor dem hinteren Schildchen und vereinigen sich ziemlich bald.

Der Darm bildet sehr wenig Windungen (6). Der Spindelmuskel ist vollständig und inseriert hinten im Körper. Der Enddarm ist von mässiger Länge, etwa von halber Körperlänge. Die Mündung des Enddarmes liegt vor den Segmentalorganen. Der Ösophagus ist

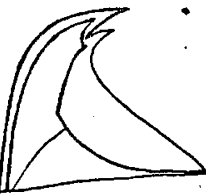

Fig. 23. Haken von A. gerouldi.

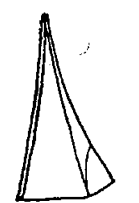

Fig. 24. Rüsseldorn von A. gerouldi.

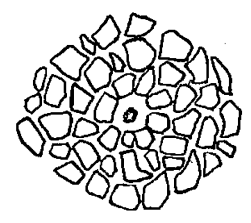

Fig. 25. Körperpapille von $A$. gerouldi. von einem einfachen kontraktilen Schlauch begleitet.

Die beiden Segmentalorgane sind etwas länger als die halbe Körperlänge und über ihre ganze Länge festgeheftet.

Vorn am Rüssel 12 fingerförmige Tentakel. Hinter diesen viele Hakenreihen. Die Haken haben zwei Spitzen und sind klein. (Fig. 23). Ihre Höhe ist 0,036 mm, ihre Breite 0,032 mm.

Am Hinterende des Rüssels stehen schmale Dornen $0,040 \mathrm{~mm}$ hoch und 0,016 mm breit. (Fig. 24).

Die Körperpapillen haben eine zentrale Öffnung, darum konzentrisch angeordnete Plättchen, die nach der Peripherie etwas grösser werden (Fig. 25).

\section{Aspidosiphon steenstrupi Diesing.}

Caracas-Bai, aus Korallen, 2. IV. '20, $1 \mathrm{Ex}$.

Wir folgen hier der Beschreibung SelenKA's: Farbe hellgelbbraun, an den Enden dunkler. Die Körpermitte ist so durchscheinend, dass man deutlich die Längsmuskeln als gesonderte, häufig anastomosierende Stränge unterscheiden kann. Über den ganzen Körper zerstreut liegen gröbere und feinere Punkte, Hautkörper, namentlich deutlich an beiden Enden; sie bestehen aus nebeneinander liegenden polygonalen Plättchen, von denen vier oder mehr in ihrer Mitte je eine Öffnung tragen. Die beiden Schilder sind je nach dem Kontraktionszustande halbkuglig gewölbt oder flach. Da am Rande des Hinterschildes die Riefen sehr ungleich und unregelmässig ausgebildet sind, so lässt sich ihre Zahl nicht genau angeben; um den Mittelpunkt herum verlaufen ein Paar feine konzentrische Ringe und in diesem Teil, der ungefähr die innere Hälfte der Scheibe einnimmt, liegen ganz unregelmässig neben einander kleine Körper von meistens ovaler Gestalt; weiter nach aussen zu werden diese Gebilde grösser, polygonal und lagern sich zu deutlich erkennbaren Feldern zusammen, deren Ränder häufig radiär gelagert sind. Das vordere Schildchen ist oft mit weissen Kalkkörnern dicht besetzt. Unmittelbar hinter seinem unteren Rande tritt der Rüssel hervor, der hinten dicht mit braunen Zähnen, vorne mit Hakenreihen bewaffnet ist. Zwischen jenen zerstreut liegen die Mündungspapillen der Hautkörper. Die Haken haben zwei Spitzen.

Die Längsmuskelstämme sind vorn stärker entwickelt als hinten, man unterscheidet hinten ca. 25 Stränge, welche so häufige Anastomosen bilden, dass vorn nur etwa 16 vorhanden sind; in der Gegend der Mündungsstellen der Segmentalorgane beginnen sie stark mit einander zu verschmelzen.

Der Retraktor setzt sich aus zwei Wurzeln zusammen, deren Basis am Anfang des hinteren Viertels der Körperlänge liegt. Sie entspringen aus dem 3. bis 7. oder 8. Längsmuskelstrange, ihr Vereinigungspunkt liegt etwa in der Mitte des Körpers.

Die Windungen der Darmspira umfassen einen starken Spindelmuskel, welcher sich am Hinterende befestigt; der Enddarm bildet drei weite Windungen und setzt sich vorn unmittelbar vor dem Anus 
an die Körperwand fest. Ein kontraktiler Schlauch ist vorhanden und einfach gestaltet. Die Segmentalorgane erstrecken sich bis zur Basis der Retraktoren und sind zur Hälfte frei. Ein Divertikel am Darm.

Fundorte von A. steenstrupi sind: Bohol Mindanas (SEmPER); Mauritius (MöBIUs); Noesa Kambangan, Südküste von Java. (C. Aurivillius); bei Koepang, Timor, in Korallen (C. Aurivillius); St. Barthélemy in Madreporen (A. v. Gö̈s); St. Thomas, Wreck Bay (C. Bovallius).

A. speciosus Gerould und A. spinoso-scutatus Fischer, die von diesen Gegenden bekannt sind, waren' nicht in dieser Sammlung vorhanden.

\section{ECHIUROIDEA.}

Von Echiuriden waren nur zwei Thalassema-Arten vorhanden: Thalassema baronii Greeff und eine neue Art: Thalassema kefersteini.

Thalassema baronii Greeff.

Spaansche Haven: 17. IV. '20, 9 Ex.; 20. V. '20, 4 Ex.

Die Körperlänge der Spiritusexemplare war etwa $3 \mathrm{~cm}$, der Kopflappen betrug etwa die Hälfte dieser Länge.

Die Papillen stehen in ziemlich deutlichen Querreihen und zwar am hintersten Teil dicht nebeneinander, weiter am Mittelkörper liegen zwischen den Querreihen grosser Papillen ein oder zwei Reihen kleineren. Vorn am Körper verliert sich die Querreihenstellung allmählich.

Auch der Kopflappen besitzt Papillen, aber nur kleinere, welche keine regelmässige Anordnung in Reihen zeigen.

Die Muskulatur ist in 19 Längsbündel gesondert, die ganz vorn am Körper sehr flach und dadurch schwer zu erkennen sind.

Die beiden Haken sind klein und stehen weit nach vorn. Der gebogene Teil ist braun gefärbt, übrigens goldglänzend.

Es sind zwei Paar Segmentalorgane vorhanden mit spiralig gewundenen innern Öffnungen. Die Analschläuche sind lang und haben kurze Verzweigungen.

Fundorte von Th. baronii sind: Kanarische Inseln (GREeF); Bahia (SHIPLEY); Loyalty Islands; Christmas Island; Tortugas, Westindien; Bird-Key Riff; St. Thomas Sound; Timor (Siboga-Expedition).

Thalassema kefersteini nov. sp.

Spaansche Haven, 16. IV.'20, viele Ex.

Bei den in Formol konservierten Tieren ist die Körperlänge etwa $25 \mathrm{~mm}$, der Rüssel $12 \mathrm{~mm}$.

Die Haut erscheint bei den konservierten Tieren ganz durchscheinend, sowohl die des Körpers als des Rüssels, sodass die Längsmuskelstränge, der Darm und der Bauchnervenstrang deutlich durch den Hautmuskelschlauch erkennbar sind.

Die zwei Hakenborsten sind goldglänzend. Nicht nur der stark gebogene Vorderteil ist ausgestülpt, sondern es ragt auch der gerade Teil weit hervor. Im ganzen ist der Haken $2 \mathrm{~mm}$ lang, wovon etwa ein Fünftel auf den eigentlichen gebogenen Teil kommt.

Nur am Vorderende und am Hinterende des Körpers stehen ziemlich dicht gedrängte kleine Papillen.

Die Haut ist dünn und hell; die Längsmuskulatur bildet 18 gesonderte Bündel. Die Raum zwischen zwei Bündeln ist nur ein Drittel der Breite der Muskelstränge.

Auf der Höhe der Hakenborsten, unmittelbar neben dem Bauchnervenstrang liegt ein Paar grosser Segmentalorgane, die keine spiraliggewundenen Ausläufer besitzen. Die Analschläuche sind gerade und lang, von etwas mehr als halber Körperlänge und hinten durch Mesenterien befestigt. 


\section{LITERATURVERZEICHNIS,}

1. Augener (1903), Beiträge zur Kenntnis der Gephyreen. Archiv f. Naturg., 69. Jahrg., Bd. 1.

2. Collin (1892), Gephyreen gesammelt von Stabsarzt Dr. Sander auf der Reise S. M. S. Adalbert. Archiv für Naturg., 58. Jahrg., Bd. 1.

3. Cú́Not (1902), Echiuriens et Sipunculiens d'Arcachon. Bulletin de la Societé scientifique d'Arcachon, Station biologique.

4. FISCHER, W. (1892), Übersicht der von Herrn Dr. Fr. Stuhlmann auf Sansibar und an den gegenüberliegenden Festlandsküste gesammelten Gephyreen. Jahrb. der Hamburg. Wissensch. Anstalten, IX, 2.

5. - - (1895), Die Gephyreen des Naturhistorischen Museums zu Hamburg. Abh. d. Naturw. Vereins Hamburg, Bd. 13.

6. - - (1914), Weitere Mitteilungen über die Gephyreen des Naturhistorischen Museums zu Hamburg. Jahrb. d. Hamb. Wissensch. Anstalten, Bd. 31, 2. Beiheft.

7. - - (1914), Gephyrea. Beiträge zur Kenntniss der Meeresfauna Westafrikas, herausg. von W. Michaelsen, Hamburg.

8. _- (1916), Die Gephyreenausbeute der Deutschen Tiefsee-Expediton (1898-1899). Vorläuf. Mitt.. Zool. Anz., Bd. 48

9. - (1919), Gephyreen der Südwestküste Australiens. Zool. Anz., Bd. 50.

10. - - (1919), Über die Gattung Lithacrosiphon, eine neue Sipunculiden-Gattung. Zool. Anz., Bd. 50.

11. - - (1920), Aspidosiphon pygmaeus, eine neue Gephyree aus Juan Fernandez. The Natural History of Juan Fernandez and Easter Island. Vol. III, herausg. von Dr. Carl Skottsberg.

12. _- (1921), Gephyreen der antarktischen und subantarktischen Meere. Deutsche Südpolarexp. 1901-1903, Bd.XVI, Zool. VIII.

13. - - (1921), Gephyreen. Results of Dr. E. Mjöberg's Sw. Scient. Exp. to Australia 1910-1913. Kungl. Sv. Vet. Ak. Handl. Bd. 61, no 8.

14. - - (1922), Gephyreen des Reichmuseums zu Stockhoim. Arkiv för Zoologi, Bd. 14, nº 19.

15. - - (1922), Westindische Gephyreen. Zool. Anzeiger, Bd. 55, nr. 1/2.

16. Gerould (1913), The Sipunculids of the eastern coast of North-America. Proc. of the U. S. Nat. Mus., Vol. 44, Washington.

17. HÉRUBEL (1908), Expédition Antarctique française 1903-1905, Vers et Brachiopodes.

18. HORST (1881), Die Gephyrea, gesammelt während der zwei ersten Fahrten der „Willem Barents”, I und II.

19. - (1898), Aspidosiphon cylindricus. Notes from the Leyden Museum, Vol. 20.

20. HoRst, C. J. v. D. (1924), Narrative of the Voyage and short description of localities, Bijdragen tot de kennis der Fauna van Curaçao. Bijdragen tot de Dierk., uitgeg. door het Kon. Zool. Gen. Natura Artis Magistra, 23e Afl.

21. IKEDA (1904), The Gephyrea of Japan. Journal of the Coll. of Sc. Imp. Univ. Tokyo, Vol. 20.

22. - - (1907), On three new and remarkable species of Echiuroids. Journal of the Coll. of Sc. Imp. Univ. of Tokyo, Vol. 21.

23. KEFERSTEIN (1865), Beiträge zur anatomischen und systematischen Kenntniss der Sipunculiden. Zeitschr. f. Wissensch. Zool., Bd. 15.

24. - - (1867), Über einige amerikanische Sipunculiden. Zeitschr. f. Wissensch. Zool., Bd. 17.

25. LANCHESTER, W. F. (1905), The marine Fauna of Zanzibar and British East-Africa, und, On a collection of Sipunculids made at Singapore and Malacca. Proc. of the Zool. Soc. of London, 1905, Vol. 1.

26. Michaelsen (1889), Die Gephyreen von Süd-Georgien. Jahrb. d. Hamb. Wiss. Anst. 6.

27. Selenka, E. (1883), Die Sipunculiden, eine systematische Monographie. C. Sempers Reisen im Archipel der Philippinen. Wissensch. Res., Bd. 4, Wiesbaden.

28. Shipley, A. (1890), On Phymosoma varians. Quarterly Journal of Microscopical Science. Vol. 31.

29. - - (1891), On a new species of Phymosoma with a Synopsis of the Genus and some account of its geographical distribution. Quart. Journ. of Micr. Sc., Vol. 32.

30. - (1892), On Ochnesoma Steenstrupii. Quart. Journ. of Micr. Sc. Vol. 33.

31. - (1898), Report of the Gephyrean worms collected by Mr. Gardiner at Rotuma and Funafuti. Proc. of the Zool. Soc. of London.

32. - (1899), A Report of the Sipunculoidea collected by Dr. Willey at the Loyalty Islands and New Britain. Willeys Zool. Res., P. 2 .

33. - - (1899), Notes on a collection of Gephyrean worms found at Christmas Island by C. W. Andrews. Proc. of the Zool. Soc. of London. 
34. Shipley, A. (1899), On a collection of Echiurids from the Loyalty Islands, New Britain and China Straits with an attempt to revise the group and to determine its geographical range. Willeys Zool. Res., Part. III.

35. - - (1902), Echiuroidea, und, Sipunculoidea, with an account of a new genus Lithacrosiphon. The Fauna and Geography of the Maldive and Laccadive Archipelagoes, ed. by J. St. Gardiner, Vol. I, Part II, Cambridge.

36. Sluiter, C. PH. (1884), Beiträge zur Kenntniss der Gephyreen aus dem Malayischen Archipel. Natuurk. Tijdschrift v. Nederl. Indië, Bd. 43.

37. - - (1886), Beiträge zur Kenntnis der Gephyreen aus. dem Malayischen Archipel. Natuurk. Tijdschrift v. Nederl. Indië, Bd. 45.

38. - - (1898), Beiträge zur Kenntnis der Fauna von Süd-Afrika. Zool. Jahrb. Abt. f. Syst., Bd. 11.

39. - - (1900), Gephyriens prov. des camp. de l'Hirondelle et de la Princesse Alice 1886-1887. Res. des Camp. Scient, par Albert, prince de Monaco, Fasc. 15.

40. - - (1902), Die Sipunculiden und Echiuriden der Siboga-Expedition. Uitkomsten Siboga-Expeditie XXV, herausgegeben von Dr. Max Weber.

41. SoutheRn (1913), Gephyrea of the coasts of Ireland. Fisheries, Ireland, Sci. Invest., 1912, III, (1913).

42. SPEnGel (1912), Einige Organisationsverhältnisse von Sipunculus-Arten und ihre Bedeutung für die Syst. dieser Tiere. Verh. d. Deutsch. Zool. Ges. Halle.

43. -- (1913), Zur Organisation und Systematik der Gattung Sipunculus. Verhandl. der Deutschen Zool. Gesellsch. auf der 23sten Jahresversammlung zu Bremen. 